\title{
La información educativa y \\ su gestión a través de Internet
}

\author{
María Esther del Moral Pérez \\ Universidad de Oviedo \\ Departamento de Ciencias de la Educación
}

\subsection{Resumen}

La gestión de la información a través de Internet es todavía muy incipiente, se echa de menos la existencia de instrumentos que ayuden a discriminar aquellos recursos útiles de entre ese ingente volumen de información disponible. Se están empezando a desarrollar aplicaciones al estilo de catálogos, revistas electrónicas, colecciones de recursos categorizados por temas, y en general, herramientas de búsqueda que contribuyan a recuperar determinado tipo de información de forma más sencilla e inmediata. Por otro lado, aprender con la mediación de las nuevas tecnologías es el nuevo reto que se plantea a la actividad docente. El individuo debe adquirir los instrumentos cognitivos y operacionales que le permitan desenvolverse en una sociedad marcada por el impacto de la tecnología en la que la incapacidad o falta de habilidad para utilizar las herramientas tecnológicas puede conducir irremediablemente a la marginación. (Autor)

Palabras clave: Tecnología de la Información. Explotación didáctica. Recursos Educativos. Formación docente. Internet.

\subsection{Abstract}

The educational possibilities of the Internet are unquestionable. It can have many applications in different fields such as distance education or the elaboration of interactive didactic material using the existing resources in the Internet. However, there is an absence of tools able to help in identifying useful resources within the huge volume of available information. Some applications are being developed to fill this gap - which contribute to retrieve information in an easy and immediate way-: catalogues, electronic magazines, collections of resources classified by topics and browsing tools. Learning to use these New Technologies is the challenge that teaching activities should now address. The individual must acquire the cognitive and operative tools that will allow him/her to perform within a society marked by the impact of technology. The inability or

Scire. 4 : 2 (jul.-dic. 1998) 35-46. 
the lack of expertise in using technological tools may lead to irretrievable margination. (Author)

Keywords: Information technology. Educative resources. Training teachers. Internet.

\section{Introducción}

El desarrollo de las TIC (Tecnologías de la Información y la Comunicación) está contribuyendo a configurar nuestra sociedad y nuestra cultura. Cada vez somos más conscientes de la dependencia que la integración de los recursos técnicos está generando en el quehacer humano.

La implementación de las TIC ha ocasionado numerosos cambios tanto en los procesos cognitivos humanos, como en la asimilación de una nueva escala axiológica, como en la aceptación de novedosas fórmulas de interacción humana, etc. Todo ello hace nos cuestiona por las implicaciones que estas TIC están comenzando a tener en el ámbito educativo.

Las innovaciones tecnológicas definen, en gran medida, nuestro horizonte puesto que conforman y limitan las condiciones de lo que es posible y lo que es tan solo imaginable (Zuboff, 1988). No se puede olvidar que si los descubrimientos y avances tecnológicos son importantes, sus aplicaciones aun lo son más. También es preciso subrayar que del uso que se haga de dicha tecnología, dependerá la consideración de la misma desde una perspectiva ético-educativa. Si adoptamos una visión optimista, podemos ver cómo la explotación que de las TIC se está haciendo está contribuyendo a mejorar la calidad de vida de las personas, aunque no del mismo modo para todos. Por otro lado, no dejan de aparecer voces alertándonos frente a los riesgos que estas tecnologías entrañan. Los debates a propósito de los límites que deben establecerse frente a la utilización de estas nuevas tecnologías están creciendo sobre manera en un intento por preservar unos valores comunes que afectan a la humanidad.

Sin embargo, y sin ánimo de agotar todas las implicaciones que aquí se han apuntado y otras que se vislumbran — puesto que desbordaría el objeto de nuestro presente trabajo-, intentaremos focalizar nuestra atención en algunas de las ventajas que presenta este nuevo canal de comunicación llamando Internet, que está integrado en ese macro-mundo de las TIC.

Así pues, inmersos en una sociedad en la que imperan los medios electrónicos y la digitalización, y en donde la información viaja más rápido que su portador, a la velocidad de la luz, se hace preciso analizar en qué medida estos nuevos medios de transmisión de datos, presentados en cualquier tipo de formato, pueden ser útiles y ponerse al servicio de personas, investigadores, entidades y corporaciones íntimamente relacionadas con el mundo educativo.

Scire. $4: 2$ (jul.-dic. 1998) 35-46. 


\section{Nuevos formatos potenciadores del proceso de enseñanza/aprendizaje}

Es un hecho que el fenómeno de la digitalización de los datos, junto a la aparición de los nuevos soportes electrónicos han generado nuevas formas de almacenar y presentar la información. Los tutoriales multimedia, las bases de datos en línea, las bibliotecas electrónicas, los hipertextos distribuidos, etc., son nuevas formas de presentar y acceder al conocimiento que superan en determinados contextos las formas tradicionales de la explicación oral, la pizarra, los apuntes y el manual. Las simulaciones de procesos junto con la representación gráfica, la integración de texto, imagen y sonido o de la navegación hipertextual han abierto nuevas vías para el diseño de instrumentos y recursos al servicio de la enseñanza. Además, este tipo de soportes está siendo utilizado de modo creciente en todos los niveles educativos (del Moral y otros, 1996).

Las herramientas de autor permiten al profesorado, además de utilizar materiales comerciales, desarrollar ellos mismos sus propios materiales, adaptados al contexto de sus estudiantes. Un ejemplo claro de la evolución a la que estamos asistiendo es cómo se están transformando las bibliotecas, y en concreto las universitarias. De simples depósitos de libros y revistas con salas de lectura anexas, están pasando a ofrecer múltiples fuentes de información electrónica. El primer paso fue la adquisición de bases de datos en CD-Rom, un soporte material para la información que hizo que los bibliotecarios más tradicionales, acostumbrados a "manejar" objetos físicos, sintieran amenazados sus puestos de trabajo o, cuando menos, modificadas en gran medida sus funciones, lo cual les ha llevado a una formación más actualizada en orden a adecuarse a estas nuevas exigencias impuestas por las tecnologías.

\section{Entornos de enseñanza/aprendizaje y de gestión de la informa- ción educativa a través de Internet}

No sólo los profesionales de la biblioteconomía y la documentación se han visto sustancialmente afectados en sus tareas profesionales como señala Graciela Caplan (1998): "hay que formar biblio-internet-arios especialistas en revisar y catalogar información”. Dentro del ámbito de la educación también se han dejado sentir los efectos de este desarrollo tecnológico, dotando de nuevos roles tanto a profesores como a estudiantes. La perspectiva tradicional en educación superior, por ejemplo, del profesor como única fuente de información y sabiduría y de los estudiantes como receptores pasivos debe dar paso a papeles bastante diferentes. La información y el conocimiento que se puede conseguir en las redes informáticas en la actualidad es casi inabordable. Cualquier estudiante universitario, utilizando la red Internet, puede conseguir información de la que su profesor tardará meses en disponer por los canales tradicionales. La misión del profe-

Scire. $4: 2$ (jul.-dic. 1998) 35-46. 
sor en entornos ricos en información es la de facilitador, la de guía y consejero sobre fuentes apropiadas de información, la de creador de hábitos y destrezas en la búsqueda, selección y tratamiento de la información. En estos entornos la experiencia, la meta-información, los "trucos del oficio", etc., son más importantes que la propia información, accesible por otros medios más eficientes.

Estos nuevos canales abren un frente en los conocimientos y destrezas importantes para el profesor que deberá utilizarlos y ayudar a que así lo hagan sus estudiantes del mismo modo que hasta hace bien poco se les impelía a que hicieran uso de los textos y revistas que se hallaban en las bibliotecas físicas, como unas herramientas más al servicio de su propia autoformación. De hecho, cada vez en más Universidades, los profesores atienden sus tutorías también por correo electrónico, tienen páginas web con los programas de sus asignaturas y las lecturas recomendadas (si están disponibles en formato electrónico) y utilizan los nuevos canales como medio de comunicación y para reforzar la interacción del grupo de estudiantes entre si (por ejemplo, a través de experiencias formativas en las que participan estudiantes y profesores de diversas universidades).

Así pues, a la vista de lo que se está exponiendo parece que las telecomunicaciones abren posibilidades metodológicas y didácticas insospechadas. Los estudiantes de una institución pueden acceder a través de las redes a datos, publicaciones, actas de congresos y simposios, etc.; pero también comunicarse con profesores y expertos de otras instituciones, con los que intercambiar opiniones y experiencias.

Sin embargo, las formas tradicionales de enseñanza (la "lectio") han resistido perfectamente los embates de la imprenta y la fotocopiadora. No sería extraño que resistieran también a las redes informáticas y los multimedia. No se trata ahora de condenar completamente una metodología de enseñanza que tiene sus virtudes. Se trata de ampliar el tipo de experiencias formativas de los estudiantes utilizando medios que van a encontrar por todas partes en su vida profesional y que forman parte de la cultura tecnológica que lo impregna todo (Cabero, Duarte y Barroso, 1998).

\section{Aprendizaje mediado por las Tecnologías de la Información y la Comunicación}

El uso de las TIC en la educación se circunscribe globalmente al ámbito de la educación primaria, secundaria, universitaria y formación de adultos. La aplicación de las nuevas tecnologías en los procesos de educación/formación de las personas nos exige la creación de nuevos modelos de aprendizaje, nuevos procedimientos y estrategias de búsqueda, organización, procesamiento y utilización de la información. 
Además, afectan a los procesos cognitivos: producen un cambio en las representaciones mentales y nos alejan de los objetos reales situándonos en un espacio de abstracción para el desarrollo de la actividad humana desconocido hasta este momento.

Aprender con la mediación de las NNTT es el nuevo reto que se plantea a la actividad docente. El individuo debe adquirir los instrumentos cognitivos y operacionales que le permitan desenvolverse en una sociedad marcada por el impacto de la tecnología en la que, en opinión de Miguel Angel Aguareles (1998), profesor de la Universidad de Barcelona, la incapacidad o falta de habilidad para utilizar las herramientas tecnológicas puede conducir irremediablemente a la marginación.

Además del acceso a fuentes documentales remotas, de la adquisición de habilidades por medio de la experimentación con simuladores cada vez más realistas y de la interactividad; las TIC ofrecen también la posibilidad de una comunicación fluida entre estudiantes y profesores, la creación de comunidades virtuales y posibilidades de trabajo más colaborativo para profesores y alumnos. Por otro lado, y desde una perspectiva psicológica positiva estas herramientas pueden estimular en el alumno una actitud activa y un mayor compromiso con su propio aprendizaje.

Desde otro punto de vista, hay que señalar su impacto en la formación a distancia. La educación a distancia no es un fenómeno novedoso. Los primeros cursos a distancia desarrollados por universidades datan de 1880. Hacia 1930 la radio tuvo su papel estelar. La Open University y otras ofrecen esta posibilidad desde hace unos cuarenta años. Recientemente, la UOC (Universidad Oberta de Calaluña) se ha establecido como universidad virtual y funciona con éxito. Internet se está usando como herramienta para la enseñanza a distancia transmitiendo contenidos y haciendo posible la comunicación sin condicionamientos espacio-temporales. En la actualidad los usos más comunes de la Internet en este campo son:

- La mensajería electrónica.

- Las aplicaciones web, en ocasiones incorporando elementos complejos como archivos audio, aplicaciones Java, etc.

- Conferencias en tiempo real.

Sin embargo, los expertos en educación a distancia recuerdan que los proyectos de enseñanza/aprendizaje a distancia se deben desarrollar teniendo en cuenta las necesidades de las personas a las que va dirigido, aspecto sorprendentemente olvidado con frecuencia, en parte porque el paradigma de base es el de un método centrado en el profesor y la materia, y no en las necesidades del alumno.

Scire. 4 : 2 (jul.-dic. 1998) 35-46. 
De ahí que se hayan de tener presentes algunos factores que influyen en el proceso de aprendizaje, y concretamente en el de los adultos, tales como:

- El concepto que tiene de sí mismo la persona que se forma.

- La disposición para aprender.

- El concepto de relevancia de la materia.

- La necesidad de aprendizaje activo.

\section{El reconocimiento del estilo personal de aprendizaje: Las opor- tunidades de aplicación y feedback)}

El aprendizaje en los adultos, tal como se va vislumblando a partir del uso de estas NNTT, es un proceso que debe ser autodirigido y en el que el rol del profesor debe ser el de un guía y facilitador. Por lo tanto, la creación de un entorno de aprendizaje a distancia efectivo debe tener especial cuidado en los siguientes aspectos:

- La planificación y el diseño instruccional.

- La comunicación interpersonal.

- Las técnicas de retroalimentación.

- Las técnicas de trabajo en equipo.

- Las estrategias de aprendizaje.

- El conocimiento de la tecnología.

- La motivación.

- Las circunstancias personales (situación, disposición y barreras culturales).

- Estilos de aprendizaje personales.

\section{El nuevo rol del profesor}

El paradigma del maestro o profesor cuya actividad docente se basa en la clase magistral es obsoleto. Las redes telemáticas pueden llegar a sustituir al profesor si éste se concibe como un mero transmisor de información ya que las redes pueden almacenar mucho más y se podrá suministrar dicha información adaptada a las necesidades particulares de cada alumno.

Pero el objetivo de la escolarización hasta la Universidad, además de la transmisión de conocimientos, es la socialización. Es por ello que se prevé que las redes, más que sustituir la educación presencial, pueden ser un complemento de la misma, permitiendo el acceso a fuentes remotas o cubriendo necesidades de escolarización en lugares en los que la distribución de la cultura sea inviable. 
Con el uso de las TIC la figura del profesor se entiende más como un tutor del proceso de aprendizaje. Con la informatización y telematización el aula se convierte en un espacio abierto e interactivo que puede conducir a una integración global, asegurando así el derecho a la educación.

El profesor adquiere la nueva responsabilidad de facilitar los medios cognitivos y operacionales para la edición y recuperación eficaz de la información, del mismo modo que la tiene sobre la enseñanza del uso de libros y otras fuentes documentales.

No se trata de reducir el problema de la función del profesor a un simple esquema de enseñar a través de Internet o no hacerlo, sino de plantear la integración de las herramientas telemáticas a la práctica docente de un modo adecuado. Este es un objetivo complejo que necesitará una inversión importante en formación y cambios en los paradigmas educativos que exigirán una intensa tarea investigadora, dado que la formación de los usos sociales de la tecnología es lenta y compleja.

\section{Profesores y alumnos editores. Gestión de la información}

La versatilidad que presentan estas NNTT hace que en la WWW cualquier persona se pueda convertir en editor y publicar a bajo coste. La información disponible sobre cualquier materia podrá ser enorme y actualizada pero no se tendrá ninguna garantía sobre su calidad y veracidad. Los contenidos de la red son tan dinámicos como vulnerables; a cambio, los contenidos en formato electrónico permiten la individualización y personalización de la información, lo cual puede, con una gestión adecuada, conducir a la optimización de los procesos de aprendizaje. Profesores y alumnos tendrán un papel activo en la creación y publicación de contenidos que propiciará la horizontalidad en las relaciones y la multidisciplinariedad a todos los niveles. El acceso a la red, tanto en el proceso de publicación como en el de recuperación de la información exige una formación específica en selección, discriminación y técnicas para publicar, además de buena capacidad lectora y de síntesis.

Sin embargo, existen algunos problemas emergentes como el de la fiabilidad/veracidad y el de la densidad de información. Por ello, puede preverse que la Internet requerirá la creación de nuevas herramientas de trabajo que faciliten el proceso de recuperación; por ejemplo, buscadores temáticos especializados o sistemas que contribuyan a ordenar el material de la red.

La red, como hemos venido señalando en trabajos anteriores (Moral, 1998) puede tener una gran utilidad como herramienta de apoyo didáctico universitario ya que permite la creación de páginas interactivas que tengan la finalidad no sólo de informar, sino de ofrecer la posibilidad de retroalimentación y a través de las

Scire. 4 : 2 (jul.-dic. 1998) 35-46. 
cuales el universitario (docente, investigador o alumno) se integre en los flujos de información. Un handicap evidente es la inexistencia de control de calidad científica y didáctica. Además, la falta de garantía de permanencia de las publicaciones da a la red un valor relativo como referencia bibliográfica para la realización de trabajos académicos, ya que no se puede asegurar la comprobación de la originalidad de los trabajos basados en esas referencias bibliográficas (VVAA, 1998).

\section{Uso educativo de la información contenida en la red.}

Acceder a los propios servidores Web es en sí mismo una experiencia educativa muy enriquecedora, pero en algunas ocasiones sucede que al intentar acceder en busca de algo específico se tropieza con otros materiales interesantes y que no son los que inicialmente se estaban buscando, provocándose en el mejor de los casos un "aprendizaje accidental" (Aguado de Cea y Moliner, 1996). Sin embargo, hay métodos para acceder a la WWW con propósitos educativos de forma estructurada, en los que cabe destacar dos aproximaciones, que en ningún caso deben ser excluyentes:

- Concebir la tecnología como un entorno cerrado de material educativo útil por sus capacidades hipermediales y de aprendizaje a distancia.

- Acceder de forma estructurada a un cuerpo abierto de materiales que no necesariamente fueron diseñados con propósitos educativos, pero que pueden ser aprovechados en exploraciones educativas guiadas.

Así pues, el uso educativo de las páginas Web es indudable. Además de poseer las peculiaridades de un sistema hipermedial, puede tener múltiples aplicaciones en distintos campos tales como la educación a distancia o la elaboración de materiales didácticos de carácter interactivo a partir de los recursos que se encuentren. Sin embargo, se echa de menos la existencia de instrumentos que ayuden a discriminar aquellos recursos útiles de entre ese ingente volumen de información disponible. Actualmente, se están empezando a desarrollar aplicaciones al estilo de catálogos, revistas electrónicas, colecciones de recursos categorizados por temas, etc., y, en general, herramientas de búsqueda que contribuyan a recuperar información de forma más sencilla e inmediata.

Intentar recopilar todos los recursos educativos que pueden ser de interés para el profesorado no es una tarea fácil debido a la gran cantidad de información disponible en la WWW, como venimos subrayando, a pesar de que ya existen algunas recopilaciones de recursos de interés educativo surgidas por iniciativa de grupos de investigadores o estudiosos de temas puntuales, a la que se han sumado algunas instituciones educativas creando sus propios enlaces y estableciendo conexión directa con aquellos recursos que consideran de interés. Otras iniciati- 
vas se encuentran más en la línea de proporcionar una guía pormenorizada de servidores Web que pueden ofrecer cierto interés a distintos sectores del profesorado, intentando presentar una breve sinopsis de sus contenidos con ánimo de rentabilizar al máximo los esfuerzos de búsqueda y el tiempo dedicado a la misma (Morlá, 1996). También se apuntan, aunque tímidamente, algunas relaciones de servidores de Web que reflejan una búsqueda específica para cada una de las materias relacionadas con el campo educativo, tal es el caso de la que recopila direcciones Web sobre el campo de la ciencia y el conocimiento científico realizada por Lowy (1997). A continuación incluimos en la tabla 1 algunas direcciones de servidores con interés educativo que se pueden encontrar en la Web (relacionados en orden alfabético).

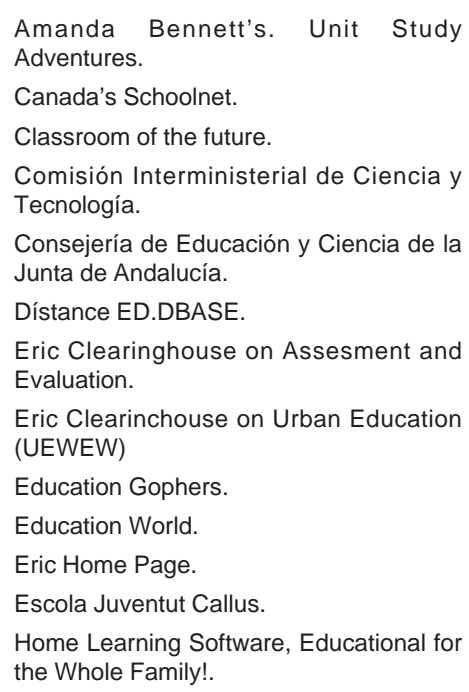



The maciazine written by and for homeschoolers.

The Sycamore tree. Homeschool and catalog.

Universidad de Sevilla.

World Wide Web Virtual Library: EDUCATION

Tabla 1. Relación de servidores con interés educativo

Por otro lado, la aplicación que hemos diseñado un grupo de investigadores de la Universidad de Oviedo (Moral y otros, 1998) pretende acercarse a la propuesta de algunos investigadores norteamericanos (Baloian, Gassner y Ultrich, 1997) en su intención de explotar el enorme potencial de la red Internet, es decir, de la enorme maraña de información, datos, documentos, imágenes, etc. que se encuentra accesible en Internet a través los servidores Webs, con objeto de integrarla de manera dinámica en los materiales educativos que se puedan utilizar en 
las aulas. En cierta medida, se trata de proporcionar una "visita guiada" (Trigg, 1988; Nielsen, 1990, p. 127); de establecer una forma de acompañar a los usuarios por un camino prefijado, de llevarles de la mano durante sus primeras experiencias de buceo en los servidores de Web. Eso sí, sin privarles de las posibilidades de navegación. Experiencias de este tipo ya han sido puestas en práctica (Nicol, Smeaton y Slater, 1995). Otras opciones parecen apostar por el diseño de índices de diversa índole: alfabéticos, jerárquicos, etc., que faciliten la localización de la información, si bien es cierto que todavía se están buscando soluciones que intenten resolver este problema.

En definitiva, todo nuestro empeño se centra en dotar al profesorado de estrategias que le permitan explotar este nuevo espacio de comunicación desde una perspectiva educativa, con objeto de que "este no lugar como lugar educativo" nos lleve a perfilar un tipo de profesor capaz de propiciar fórmulas facilitadoras del aprendizaje; de diseñar situaciones mediadas para que el aprendizaje llegue a producirse; y de generar habilidades para el asesoramiento y diagnóstico de los estudiantes, que sean más significativas que las de simple depositario de conocimientos y saberes.

\section{A modo de conclusión}

Podemos señalar para finalizar las siguientes conclusiones:

- Las redes telemáticas minimizan el despliegue de recursos económicos y de movimiento para proporcionar un vasto espacio para el intercambio de información.

- El rol del profesor será tutorizar el proceso de aprendizaje y la enseñanza dejará de ser un proceso unidireccional para ser multidireccional.

- Internet puede ser un instrumento que facilite el aprendizaje de la diversidad.

- Paulatinamente se generarán nuevas formas de concebir el mundo y la educación.

- Con las NTI el concepto de manual para la enseñanza sufrirá una metamorfosis.

- Será necesaria una buena maestría de la lectura y la síntesis.

- Habrá que formar bibliotecarios del ciberespacio o catalogadores del caos de la red y especialistas que fomenten el valor educativo del WWW.

- Mientras las tecnologías no sean de uso masivo, los beneficios serán parciales y restringidos a las realidades de los países industrializados, incrementándose así las diferencias entre países y comunidades. 
- Internet es un recurso más que facilita la búsqueda de información, el trabajo cooperativo, el conocimiento de las herramientas tecnológicas y la divulgación de la información. De ahí que, en este marco, la escuela deba fomentar el reciclaje contínuo en el conocimiento de la tecnología.

- El uso de las TIC pone en evidencia que los límites de las profesiones son cada vez más difusos y que la transdisciplinariedad va afectando progresivamente a los profesionales de cualquier campo, especialmente a los docentes que deben integrar las nuevas tecnologías a su actividad docente. La misma organización de toda la estructura educativa debe ser consecuencia de una reflexión multidisciplinar.

- En el futuro será necesario buscar el equilibrio entre el aprendizaje no presencial y el aprendizaje en el aula convencional aprovechando todos los recursos que nos proporciona la tecnología bajo la dirección y supervisión de los profesionales de la educación. Motivo por el que creemos que es necesario adoptar medidas urgentes para la capacitación de los profesores en la utilización de las TIC, y ello se debe a varios motivos. En primer lugar, que los estudios realizados sobre la formación que los profesores en general tienen para la incorporación de las diferentes tecnologías existentes en sus centros, siempre nos indican que es uno de los medios frente a los cuales los profesores autoinforman que tienen peor formación, tanto técnica, como didáctica, como referida a la producción de materiales para sus estudiantes (del Moral y otros, 1997). Y en segundo lugar, y posiblemente más importante, es que el ordenador ya no es un medio independiente, sino más bien un medio alrededor del cual giran las denominadas nuevas y avanzadas tecnologías de la información y comunicación —como por ejemplo las redes de comunicación, los multimedia o los hipertextos-que serán en torno a los cuales se produzcan los desarrollos futuros de la comunicación. En definitiva, el ordenador ha dejado de ser un medio para convertirse en un instrumento cotidiano de la vida misma.

\section{Referencias bibliográficas}

Aguado de Cea, G. ; Moliner, L. (1996). Aplicaciones educativas de la World Wide Web. // Actas de EDUTEC'95 : Redes de comunicación, redes de aprendizaje. Palma de Mayorca : Universidad, 1996. P. 103-109.

Aguareles, M.A. (1998). Informe de contenidos sobre el análisis del impacto de la telemática en el proceso de educación/formación. // I Congreso de la Publicación Electrónica. <URL=http:/www.gpd.org/maig98>.

Baloian, N. ; Gassner, K. ; Ultrich, H. (1997). Integration of External WWW-Documents into the Electronic Classroom. // World Conference on Educational Multimedia and Hypermedia, University of Calgary, Canada, June 14-19.

Scire. $4: 2$ (jul.-dic. 1998) 35-46. 
Cabero, J. ; Duarte, A. ; Barroso, J. (1998). La piedra angular para la incorporación de los medios audiovisuales, informáticos y nuevas tecnologías en los contextos educativos: la formación y el perfeccionamiento del profesorado. // Revista electrónica de Tecnología Educactiva EDUTEC. 8 (abr. 1998). <URL: http://www.uib.es /depart/gte/revelec6.htm>.

Caplan, G. (1998). Reflexiones vertidas en el Foro Telemático sobre Tecnología Educativa, mayo 1998.

Lowy, E. (1997). Las páginas Web de Internet en la educación científica. Boletín del CDL. 85 ( mayo 1997) 14-15.

Moral, M. E. del ; García, O. (1996). Las posibilidades que ofrece INTERNET a los profesionales de la educación : Investigación, Formación y Reciclaje. // I Simposio Iberoamericano sobre Redes de Comunicación para la Educación, Mar del Plata, Argentina, 6-10 de Mayo, 1996.

Moral, M. E. del ; et al. (1997). Actitudes del profesorado hacia la integración de las NNTT de la comunicación en la educación. // EDUTEC'97, 27-29 octubre, Universidad de Málaga.

Moral, M. E. del ; et al. (1998). TIMÓN : Navegation System Through Web Server with Educative Interest for the Didactic Exploitation of Sources of Resources. // EDMEDIA \& ED-TELECOM 98 : World Conference on Educational Multimedia and Hypermedia. University of Freiburg (Alemania), 20-25 June.

Morlá, M.M. (1996). Webs de interés educativo : Una guía para el profesorado. // Actas de EDUTEC'95 Redes de comunicación, redes de aprendizaje. Palma de Mayorca : Universidad, 1996. p.123-132.

Nicol, D. ; Smeaton, C. ; Slater, A.F. (1995). Footsteps : Trail-blazing the Web. // Proceedings of the Third International Conference on the World-Wide Web, April 1995, Darmstadt, Germany.

Nielsen, J. (1990). The Art of Navigating Through Hypertext. // Communications of the ACM. 33 : 3 (1990) 296-310.

Pablos, J. de ; Jiménez, J. (Coord) (1998). Nuevas Tecnologías : Comunicación Audiovisual y Educación. Barcelona : Cedecs, 1998.

Trigg, R. H. (1988). Guided tours and tabletops : Tools for communicating in a hypertex environment. // ACM Trans. Office Information Systems. 6 : 4 (1988) 389-414.

VVAA (1998). Informe de contenidos sobre el análisis del impacto de la telemática en el proceso de educación/formación. // I Congreso de la Publicación Electrónica. <URL=http:/www.gpd.org/maig98>.

Zuboff, S. (1988). In the age of the smart machine. Nueva York : Basic Books, 1988. 